\title{
Which Skin Lupus Criteria are of More Value in Determining the Disease: A Greater View with the Help of Direct Immune Fluorescent (DIF)
}

\section{Sahra Emamzadehfard ${ }^{1-3}$, Alireza Ghannadan ${ }^{1,3 *}$ and Sara Pourhassan Shamchi ${ }^{2,4}$}

${ }^{1}$ Department of Dermatopathology, Razi Dermatology Hospital, Tehran University of Medical Sciences, Tehran, Iran

${ }^{2}$ Department of Radiology, University of Pennsylvania, Philadelphia, USA

${ }^{3}$ Department of Pathology, Cancer Institute, Imam Khomeini Hospital Complex, Tehran University of Medical Sciences, Tehran, Iran

${ }^{4}$ Department of Pathology, Tabriz University of Medical Sciences, Tabriz, Iran

\begin{abstract}
Background: Lupus erythematosus (LE) includes a spectrum of auto immune disorders, involving whole body (systemic LE) in one end and is cutaneous limited LE on the other side. Cutaneous LE mainly diagnosed via clinical manifestations as well as histopathologic examination. According to different pathologic specifications, the aim of present study was to determine which histopathologic criterion is more accurate and practical in diagnosing cutaneous LE.

Material and Methods: The Samples which have been taken were from the patients with clinical manifestations of Cutaneous LE with pathologic confirmation. All patients had direct immuno fluorescent (DIF) samples that recorded. Histopathologic findings are categorized into three groups: group 1 contains epidermal changes, group 2 includes interface changes and group 3 contains dermal changes. Different histopathologic finding such as prevascular infiltration, follicular atrophy, follicular plaque, and basements membrane thickness and other changes, as well as DIF patterns, were recorded in designed questionnaires. Further analysis had been done with SPSS software version 22.
\end{abstract}

Results: Of 145 patients $(61.4 \%$ female and $38.6 \%$ male) in $58.6 \%$ DLE was the first clinical diagnosis, $23 \%$ was the second and in subsequent $4.1 \%, 1.4 \%$ and $0.7 \%$ was third, fourth and fifth diagnosis respectively. In $11 \%$ of caeses DLE was not among clinical impressions. DIF was positive in $49 \%$. As a whole, superficial perivascular and perifolicular infiltration were observed in $99 \%$ of the cases and was the commonest pathologic feature followed by basal vacuolization, peri follicular infiltration and epidermal atrophy. Other pathologic changes were observed with variable rates.

Discussion: By grouping histopathologic criterion, it seems that hyperkeratosis and thickening of Basement membrane may be major histologic criterion and peri vascular infiltration, peri follicular infiltration and eccrine gland infiltration, may be minor.

Keywords: Skin; Immune; Fluorescent; Lupus erythematous

Abbreviations: SPVIL: Superficial perivascular infiltrate of lymphocyte; DPVIL: Deep perivascular infiltrate of lymphocyte; PFIL: Perifollicular infiltrate of lymphocytes; PEIL: Perieccrine infiltrate of lymphocytes

\section{Introduction}

Genetic, hormones, and environment play altogether to give birth to lupus erythematosus (LE); a disease of autoimmune features with a more frequency in women especially in childbearing age which can present variously in different involved organs. The aforesaid disease could be manifested from a skin lesion (cutaneous lupus) to a fatal diffused type (systemic lupus erythematosus [SLE]). LE is clinically divided to three classes: systemic lupus with acute onset, sub- acute cutaneous type, and a chronic discoid form (discoid lupus erythematosus [DLE]). Occurring two to three times more often than SLE, cutaneous lupus is a variant of LE being able to bother the patients' daily life [1-15].

On the other side, DLE is characterized by well-defined erythematosus scaly patches mostly seen on face and other sun exposed areas; palmoplantar lesions are rarely seen in DLE and can be disabling to the patients especially when we know they are refractory to the conventional treatments [16-19]. There is a variant of DLE called tumid lupus erythematosus (TLE) which is recognized by excess production of mucin in derma giving the skin a brawny appearance with indurations. Interface dermal infiltration of lymphocytes involving superficial and deep perivascular areas, periappendageal sites, follicles, and epidermis are histopathological changes in DLE [20]. Lupus band test by using immunofluorescence also shows how autoantibodies of IgG, IgM, and IgA type have deposited over the dermoepidermal junction (DEJ); it is notable that its definition from a test confined to the skin lesions have expanded to all the areas on the skin of peoples with LE whether in the sun exposed or protected areas of the body [21-28].

It is mentioned that the diagnosis will come out when clinical manifestations (symptoms), serology (auto antibodies detected by sensitive rather than specific enzyme-linked immunosorbent assay [ELISA]), and pathology (histopathological changes in the skin lesions' sampling) all are correlated to each other [29]. Our study here aims to

*Corresponding author: Sahra Emamzadehfard, MD, Department of Dermatopathology, Razi Dermatology Hospital, Tehran University of Medical Sciences, Tehran, Iran, Tel: +982155159988; Fax: +982155155050; E-mail: sahra.e.fard@gmail.com

Received March 11, 2016; Accepted March 15, 2016; Published March 26 , 2016

Citation: Emamzadehfard S, Ghannadan A, Shamchi SP (2016) Which Skin Lupus Criteria are of More Value in Determining the Disease: A Greater View with the Help of Direct Immune Fluorescent (DIF). J Clin Med Genomics 4: 139. doi:10.4172/2472-128X.1000139

Copyright: (C) 2016 Emamzadehfard S, et al. This is an open-access article distributed under the terms of the Creative Commons Attribution License, which permits unrestricted use, distribution, and reproduction in any medium, provided the original author and source are credited. 
find out the prevalence of each pathologic criterion and to determine the benefits of these criteria over the other ways with a special attention to the direct immunofluorescence (DIF) test to reach a more accurate diagnosis on cutaneous lupus erythematosus particularly DLE.

\section{Methods}

The current survey has retrospective design which had been held in Razi Hospital, which is affiliated with Tehran University of Medical Sciences. All the patients with a pathologically confirmed diagnosis of skin lupus referring to Razi hospital were included in the study between 2008 and 2010. Another inclusion criterion was patients consent to enroll in the study. Pathologic samples were extracted from the medical records of patients and assessed by the faculties under the light microscopy for the histopathological features of the DLE; lupus band test was also evaluated on these and recorded as positive or negative result based on the pattern of antibodies, complement, and membrane attack complex deposition in dermo-epidermal junction to see if it is of any benefit over the other pathologic criteria in confirming a clinical diagnosis of DLE.

Data were analyzed by Statistical Package for the Social Sciences version 22 (SPSS) and cross tab software to find the relationship between each criterion; of all histopathologic features, hyperkeratosis, follicular atrophy, follicular plugging, and basements membrane thickness increasing were in a single group (group 1); superficial and deep peri vascular infiltration in another category (group 2), and perivascular, perifollicular, and perieccrine were included in a separated one (group 3). Overall results were used in bringing out a series of pathologic criteria for accurate diagnosis of cutaneous lupus. Differential diagnoses similar clinically to cutaneous LE were also assessed to find out the prevalence of their histopathological differences. Test results from DIF were also another aspect of the study which was evaluated for its prevalence to see the efficacy.

\section{Result}

Of 145 cutaneous lupus samples, $61.4 \%$ and $38.6 \%$ were belonged to the females and males, respectively. In $58.6 \%$ of the patients, DLE had been the first diagnosis associated with $23.4 \%$ in the second rank, $4.1 \%$ in the third, $1.4 \%$ fourth, $0.7 \%$ fifth, and in $11 \%$ of the cases, it had not been among the diagnoses. Also DIF was reported positive in $49 \%$ of the cases with $25 \%$ IgG, $33 \%$ IgM, and $42 \%$ C 3 all deposited in the dermo- epidermal junction Table 1 . The differential diagnoses are shown in table 2 . Also in $36.6 \%$ of the cases, the presenting symptom was plaque, $16.6 \%$ papule, $15.9 \%$ erythematosus lesion, $10.3 \%$ patch, $5.5 \%$ alopecia, $4.8 \%$ pigmentation, $2.1 \%$ nodule, and other $0.7 \% \mathrm{scar}$, rash, blister, macula, erosion, hypopigmentation, and vesicles (Table 2).

The frequency of histopathologic features in three histopathologic groups is summarized in Tables 3-5 regardless of the sequence they have appeared at the field. The most common finding of histopathology in our study was SPVIL that was the same with other articles.

\begin{tabular}{|l|c|}
\hline Demographic data & No. of cases (\%) \\
\hline Sex & \\
\hline Man & $56(38.6)$ \\
Woman & $89(61.4)$ \\
\hline Age & 23 \\
\hline Range & 24 \\
\hline Mean & 1 \\
\hline Site & \\
\hline
\end{tabular}

Table 1: Demographic data of different cases.

\begin{tabular}{|l|c|}
\hline Type of antibody & No. of cases (\%) \\
\hline $\lg G$ & $71 / 145(49)$ \\
\hline $\lg M$ & $48 / 145(33)$ \\
\hline C3 & $60 / 145(42)$ \\
\hline
\end{tabular}

Table 2: Amount of antibody.

\begin{tabular}{|l|c|}
\hline Epidermal changes & No. of cases (\%) \\
\hline Epidermal atrophy & $107(73.8)$ \\
\hline Hyperkeratosis & $91(62.8)$ \\
\hline Follicualr plugging & $84(57.9)$ \\
\hline Parakeratosis & $45(31)$ \\
\hline Spongiosis & $31(21.4)$ \\
\hline Epidermal hyperplasia & $19(13.1)$ \\
\hline Lymphoexocytosis & $18(12.4)$ \\
\hline
\end{tabular}

Table 3: Epidermal changes data.

\begin{tabular}{|l|c|}
\hline Dermoepidermal changes & No. of cases (\%) \\
\hline Basal vacuolation & $120(83.4)$ \\
\hline Lichenoid infiltration & $95(65.5)$ \\
\hline Melanin incontinency & $80(55.2)$ \\
\hline Civatte bodies & $26(17.0)$ \\
\hline BM thickening & $17(11.7)$ \\
\hline
\end{tabular}

Table 4: Dermoepidermal changes data.

\begin{tabular}{|l|c|}
\hline Dermal changes & No. of cases (\%) \\
\hline SPVIL & $137(94.5)$ \\
\hline PFIL & $117(80.7)$ \\
\hline DPVIL & $104(71.7)$ \\
\hline PEIL & $101(69.7)$ \\
\hline Follicular atrophy & $67(46.2)$ \\
\hline Mucin deposition & $45(31)$ \\
\hline
\end{tabular}

Table 5: Dermal changes data.

\begin{tabular}{|l|c|}
\hline Histopathology combination (\%) & Percentage \\
\hline SPVIL (94.5) + BM thickening (11.7) & $100 \%$ \\
\hline SPVIL (94.5) + PFIL (80.7) & $99 \%$ \\
\hline SPVIL (94.5) + F. plugging (57.9) & $96.4 \%$ \\
\hline DPVIL (71.7) + PEIL (69.7) & $95 \%$ \\
\hline
\end{tabular}

Table 6: Histopathology combination (\%) data.

We analyzed data using SPSS and cross tab software to connect the features of three groups to each other to see which combination can bring a more accurate diagnosis. The most powerful associations of histopathologic findings were SPVIL and BM thickening (100\%), SPVIL and PFIL (99\%), follicular plugging and SPVIL (96.4\%), DPVIL and PEIL (95\%), PFIL and PEIL (90\%). These data are summarized in Table 6.

Other analysis was done to find out the relations between these categories; it showed that hyperkeratosis and superficial perivascular infiltration are seen in 94.5\%, follicular atrophy and superficial perivascular infiltration in $79 \%$, follicular plaque with superficial perivascular infiltration in $96.4 \%$ while with reflective in $89.2 \%$, superficial perivascular infiltration and basements membrane thickness increasing in $100 \%$, basements membrane thickness increasing and deep perivascular infiltration in $88 \%$, and deep perivascular infiltration with superficial perivascular, perifolicular, and paracrine infiltration in $95.10 \%, 87.5 \%$ and $90.9 \%$ respectively. Deposition of C3 in dermoepidermal junction in combination with basements membrane thickness increasing, were associated with the highest rate of positive predictive value for the diagnosis of SLE. 
Citation: Emamzadehfard S, Ghannadan A, Shamchi SP (2016) Which Skin Lupus Criteria are of More Value in Determining the Disease: A Greater View with the Help of Direct Immune Fluorescent (DIF). J Clin Med Genomics 4: 139. doi:10.4172/2472-128X.1000139

Page 3 of 4

\section{Discussion}

Our results showed a different proportion of gender in the evaluated cases; $61.4 \%$ of female to $38.6 \%$ male in comparison to a $83 \%$ to $17 \%$ proportion in other sources. The most prevalent finding was perivascular infiltration which is in absolute correlation with other studies. When looking from one aspect to the frequency of the histopathologic features and consider them with one direction, hyperkeratosis and basement membrane thickness increasing altogether in association with one or two other features of this category, superficial and deep perivascular infiltration in association with one or two other features of other categories, and superficial perivascular, perifolicular, and paracrine infiltration have all more sensitivity to determine cutaneous lupus pathologically; while, another analytical look told a different story.

The research which has been done by Tiao et al tried to use the American College of Rheumatology (ACR) and Systemic Lupus International Collaborating Clinics (SLICC) criteria to determine the diagnosis of systemic lupus erythematosus in patients with subacute cutaneous lupus erythematosus. They concluded that most patients with cutaneous SLE who formally meet criteria for SLE do so based on the laboratory and mucocutaneous criteria. Neither the ACR nor SLICC criteria distinguish patients with subacute cutaneous lupus erythematosus (SCLE) and major internal disease from patients with SCLE without major internal disease. They concluded that renal disease and central nervous system disease were no more frequent in patients with SCLE who met SLE criteria than in patients with SCLE who did not. Most patients with SCLE who formally meet criteria for SLE do so based on the laboratory and mucocutaneous criteria. Neither the ACR nor SLICC criteria distinguish patients with SCLE and major internal disease from patients with SCLE without major internal disease [30].

Most previous studies comparing patients with SCLE versus those with only SLE have demonstrated that patients with SCLE are less likely to have severe renal and central nervous system disease, suggesting that SCLE differs from SLE by involving less internal organ disease [31-35].

In the survey which has done by Merklen et al, they analyzed 22 SLE patients with epidermal detachment. They revealed two main pathomechanisms: a classic SLE interface dermatitis, which can be hyperacute and lead to toxic epidermal necrolysis (TEN)-like skin detachment; and a neutrophilic dermatosis, with tense vesicles and/or blisters, including classic bullous SLE [36].

We can concluded from the previous researches that in patients with LE, we should definitely separate bullous lesions/loss of epidermis occurring in the setting of an interface dermatitis, from those occurring as a consequence of a neutrophilic dermatosis. The latter usually respond to dapsone, and can or cannot be immunopathologically characterized, whereas the former can either be a bullous variant of classic lupus lesions or, rarely, a life-threatening TEN-like acute dermatosis. Classic "bullous LE" is a dapsone-sensitive neutrophilic dermatosis, which probably encompasses different autoimmune bullous diseases. In the series reported here, the patients with neutrophilic bullous LE were those who had most frequently experienced associated significant renal involvement [36].

As these analytical combinations show, we can mention that superficial perivascular infiltration along with hyperkeratosis, and basements membrane thickness increasing and deep perivascular infiltration, perifolicular infiltration and paracrine infiltration could be considered as cutaneous lupus diagnostic criteria.

\section{References}

1. Tebbe B, Orfanos CE (1997) Epidemiology and socioeconomic impact of skin disease in lupus erythematosus. Lupus 6: 96-104.

2. Provost TT (1994) The relationship between discoid and systemic lupus erythematosus. Arch Dermatol 130: 1308-1310.

3. Kalunian KC (1997) Definition, classification, activity, and damage indices, In Wallace DJ, Hahn BD and Dubois' Lupus Erythematosus. ( $5^{\text {th }}$ edn $)$, Baltimore, Williams \& Wilkins.

4. Tan EM, Cohen AS, Fries JF, Masi AT, McShane DJ, et al. (1982) The 1982 revised criteria for the classification of systemic lupus erythematosus. Arthritis Rheum 25: 1271-1277.

5. Sontheimer RD, Thomas JR, Gilliam JN (1979) Subacute cutaneous lupus erythematosus: a cutaneous marker for a distinct lupus erythematosus subset. Arch Dermatol 115: 1409-1415.

6. Gilliam JN, Sontheimer RD (1981) Distinctive cutaneous subsets in the spectrum of lupus erythematosus. J Am Acad Dermatol 4: 471-475.

7. Rowell NR, Goodeld MJD (1992) The connective tissue diseases. In: Champion $\mathrm{RH}$, Burton JL, Ebling FJG, Text book of Dematology. (5th edn), London: Blackwell Scientific Publications.

8. Bangert JL, Freeman RG, Sontheimer RD, Gilliam JN (1984) Sub-acute cutaneous lupus erythematosus and discoid lupus erythematosus: comparative histological findings. Arch Dermatol 120: 332

9. Jerdan MS, Hood AF, Moore GW, Callen JP (1990) Histopathologic comparison of the subsets of lupus erythematosus. Arch Dermatol 126: 52-55.

10. David-Bajar KM1 (1993) Subacute cutaneous lupus erythematosus. J Invest Dermatol 100: 2S-8S.

11. Watanabe T, Tsuchida T (1995) Classification of lupus erythematosus based upon cutaneous manifestations. Dermatological, systemic and laboratory findings in 191 patients. Dermatology 190: 277.

12. Magro C, Crowson AN, Harrist TJ (1996) The use of antibody to C5b-9 in the subclassification of lupus erythematosus. Br J Dermatol 134: 855-862.

13. Chlebus E, Wolska H, Blaszczyk M, Jablonska S (1998) Subacute cutaneous lupus erythematosus versus systemic lupus erythematosus: diagnostic criteria and therapeutic implications. J Am Acad Dermatol 38: 405.

14. Prsytowsky SD, Gilliam JN (1975) Discoid lupus erythematosus as part of a larger disease spectrum. Correlation of clinical features with laboratory findings in lupus erythematosus. Arch Dermatol 111: 1448.

15. Furukawa F, Tokura Y, Matsushita K, Iwasaki-Inuzuka K, Onagi-Suzuki K, et al. (1996) Selective expansions of T cells expressing $V$ beta 8 and $V$ beta 13 in skin lesions of patients with chronic cutaneous lupus erythematosus. J Dermatol 23: 670-676.

16. Prystowsky SD, Herndon JH Jr, Gilliam JN (1976) Chronic cutaneous lupus erythematosus (DLE)--a clinical and laboratory investigation of 80 patients. Medicine (Baltimore) 55: 183-191.

17. Sontheimer RD, Provost TT (1996) Lupus erythematosus. In: Sontheime $\mathrm{RD}$, Provost TT, editors. Cutaneous manifestations of rheumatic diseases, Baltimore. William \& Wilkins 18-21.

18. Callen JP (1982) Chronic cutaneous lupus erythematosus. Clinical, laboratory, therapeutic, and prognostic examination of 62 patients. Arch Dermatol 118 412-416.

19. Callen JP (1985) Intralesional triamcinolone is effective for discoid lupus erythematosus of the palms and soles. J Rheumatol 12: 630-633.

20. Crowson AN, Magro C (2001) The cutaneous pathology of lupus erythematosus: a review. J Cutan Pathol 28: 1-23.

21. al-Suwaid AR, Venkataram MN, Bhushnurmath SR (1995) Cutaneous lupus erythematosus: comparison of direct immunofluorescence findings with histopathology. Int J Dermatol 34: 480-482.

22. Burnham TK, Neblett TR, Fine G (1970) Immunofluorescent "band" test fo lupus erythematosus. II. Employing skin lesions. Arch Dermatol 102: 42-50.

23. Harrist TJ, Mihm MC (1979) Cutaneous immunopathology. The diagnostic use of direct and indirect immunofluorescence techniques in dermatologic disease. Hum Pathol 10: 625-653. 
Citation: Emamzadehfard S, Ghannadan A, Shamchi SP (2016) Which Skin Lupus Criteria are of More Value in Determining the Disease: A Greater View with the Help of Direct Immune Fluorescent (DIF). J Clin Med Genomics 4: 139. doi:10.4172/2472-128X.1000139

24. Harrist TJ, Mihm MC Jr (1980) The specificity and clinical usefulness of the lupus band test. Arthritis Rheum 23: 479-490.

25. Provost TT (1981) Lupus band test. Int J Dermatol 20: 475.

26. Smith CD, Marino C, Rothfield NF (1984) The clinical utility of the lupus band test. Arthritis Rheum 27: 382-387.

27. Jordon RE (1985) Lupus band test: clinical applications. Clin Dermatol 3: 113122.

28. Magro CM, Crowson AN (1999) The application of immuno fluorescence testing in diagnostic dermatopathology. In: Cock-erell CJ, (1st edn). Advances in Dermatology. St. Louis: Mosby.

29. Mutasim DF, Adams BB (2000) A practical guide for serologic evaluation of autoimmune connective tissue diseases. J Am Acad Dermatol 42: 159-174.

30. Tiao, Janice, Feng R, Carr K, Okawa J, Werth VP (2016) Using the American College of Rheumatology (ACR) and Systemic Lupus International Collaborating Clinics (SLICC) criteria to determine the diagnosis of systemic lupus erythematosus (SLE) in patients with subacute cutaneous lupus erythematosus (SCLE). Journal of the American Academy of Dermatology 15 : S0190-9622
31. Black DR, Hornung CA, Schneider PD, Callen JP (2002) Frequency and severity of systemic disease in patients with subacute cutaneous lupus erythematosus. Arch Dermatol 138: 1175-1178.

32. Lopez-Longo FJ, Monteagudo I, Gonzalez CM, Grau R, Carreno L (1997) Systemic lupus erythematosus: clinical expression and anti-Ro/SSea response in patients with and without lesions of subacute cutaneous lupus erythematosus. Lupus 6: 32-39.

33. Chlebus E, Wolska H, Blaszczyk M, Jablonska S (1998) Subacute cutaneous lupus erythematosus versus systemic lupus erythematosus: diagnostic criteria and therapeutic implications. J Am Acad Dermatol 38: 405-412.

34. Beutner EH, Blaszczyk M, Jablonska S (1991) Studies on criteria of the European Academy of Dermatology and Venerology for the classification of cutaneous lupus erythematosus. I. Selection of clinical groups and study factors. Int J Dermatol 30: 411-417.

35. Parodi A, Rebora A (1997) ARA and EADV criteria for classification of systemic lupus erythematosus in patients with cutaneous lupus erythematosus. Dermatology 194: 217-220.

36. Merklen-Djafri, Carine L (2015) Blisters and Loss of Epidermis in Patients with Lupus Erythematosus: A Clinicopathological Study of 22 Patients. Medicine 94: e2102. 